\title{
Gossypiboma can remain asym ptomatic for a long time
}

\author{
Padam Raj Pant ${ }^{1,2}$, PhD; Asmita Ghimire ${ }^{1}$, MD; Nilam Subedi ${ }^{1}$, MD; \\ Samriddha Raj Pant², MBBS; Asmita Shrestha ${ }^{1}$, PCL Nursing \\ ${ }^{1}$ Department of Gynecology \& Obstetrics, Grande International Hospital, Kathmandu, Nepal \\ ${ }^{2}$ Department of Gynecology \& Obstetrics, Institute of Medicine, Tribhuvan University Teaching Hospital, \\ Kathmandu, Nepal
}

Corresponding author

Padam R Pant, PhD

Email: padam1629@gmail.com

Received 14 Oct 2018

Accepted 4 Dec 2018

\section{ABSTRACT}

Gossypiboma, a retained foreign body after surgery, is a rare complication. It appears years after surgery, has nonspecific symptoms, and is usually identified on imaging sequences. We present here an asymptomatic case of a 32 years old lady with a history of laparotomy done two years earlier. She was initially misdiagnosed clinically and radiologically as hydatid cyst of pelvis. During re-surgery, foreign body was detected. Postoperative period was uneventful. To prevent these types of complications, we have to follow the main principle of surgery in all procedures i.e. ensuring a proper count of surgical materials before and after surgery.

Keywords: Gossypiboma, Uterus

\section{Introduction}

A gossypiboma refers to a foreign object such as sponge, gauze left accidently behind in body cavity during operation. It is derived from Latin word "gossypium" (cotton) and swahili word "boma" (place of concealment) ${ }^{1}$. It is also known as textiloma or cottonoid. Patient can present either acutely or remain asymptomatic for years in chronic state. Radiological investigations may aid in diagnosis. Definitive diagnosis and management is through operative procedure. Principle of counting the surgical instruments and sponges is an effective preventable measure.

\section{Case Report}

A 32 years regularly menstruating woman during her regular checkup was diagnosed incidentally to have a big complex pelvic mass on ultrasonogram, suspected as hydatid cyst. She was asymptomatic. Investigations done showed total count being $16000 / \mathrm{mm}^{3}$ and ESR $26 \mathrm{~mm} / \mathrm{hr}$. Except CA125 $(=89 \mathrm{ng} / \mathrm{ml})$, all her tumor markers (CEA, AFP, LDH, $\beta$-hCG) were within normal range. Magnetic resonance imaging (MRI) done was in favor of hydatid cyst of pelvis.

She was a para second lady; both were normal vaginal deliveries in hospital. She gave history of laparotomy done two years back for ectopic pregnancy. She had history of severe abdominal pain then. According to the patient, it was negative laparotomy (there was no ectopic pregnancy). Her post-operative period then was uneventful.

With preoperative diagnosis of hydatid cyst of pelvis, counseling for surgery was done. During laparotomy the findings were surprising. Though it was a difficult approach, after meticulous adhesiolysis a cystic encapsulated mass was found, which ruptured during the dissection releasing straw colored fluid. A net like solid structure was visible. It was a retained gauze. Postoperatively she had no issues and recovered uneventfully.

\section{Discussion}

Gossypiboma remains a dreadful experience. Incidence is 12 per 100,000 operations ${ }^{2}$. However due to legal issues its exact incidence is still 


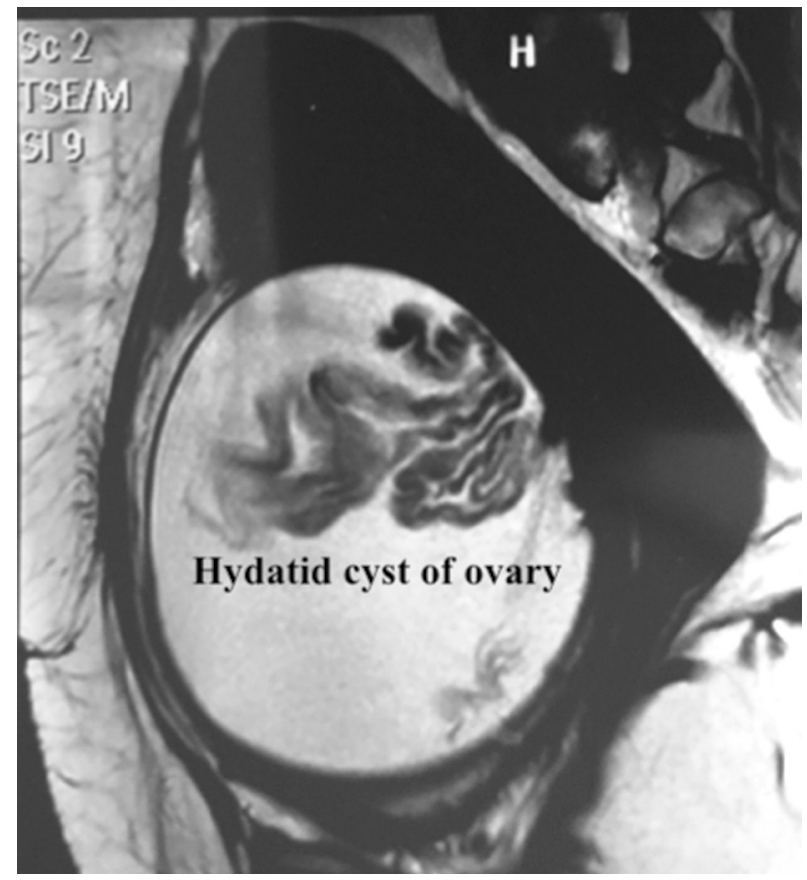

Figure 1: MRI showing hydatid cyst of ovary

unknown. Regarding surgical procedures, it occurs mainly in emergency operations. It is also seen in surgeries of paraspinal muscles, intrathoracic regions, legs, shoulder and pericardial space ${ }^{3,4}$. Obesity of the patients, change in the surgical team in between procedures, improper and hurried counting of surgical goods at the end of procedures, long operations, inexperienced and inadequate staff numbers also contribute to the issue ${ }^{5}$.

Usually patients present in two forms: Symptomatic and asymptomatic. Symptoms arise due to the exudative reaction leading to abscess formation. Patients complain of pain abdomen, abdominal distension, fever, nausea and vomiting. Some remain asymptomatic or present with pseudo tumor syndrome ${ }^{6}$. In our case also, the patient was asymptomatic and diagnosed to have hydatid cyst of pelvis. This could be because of the fibrinous reaction that occurred leading to encapsulation of the foreign body mimicking a tumor.

In a country like ours where there are no radiolabeled sponges, it is very difficult to give a definitive diagnosis preoperatively. Radiological examination like ultrasonogram (USG), computerized tomography scan (CT scan) and MRI are usually done ${ }^{1}$. However, chronic cases like the one reported in this article do not show definite clinical and radiological signs. Patient had no symptoms and MRI favored hydatid cyst of ovary.

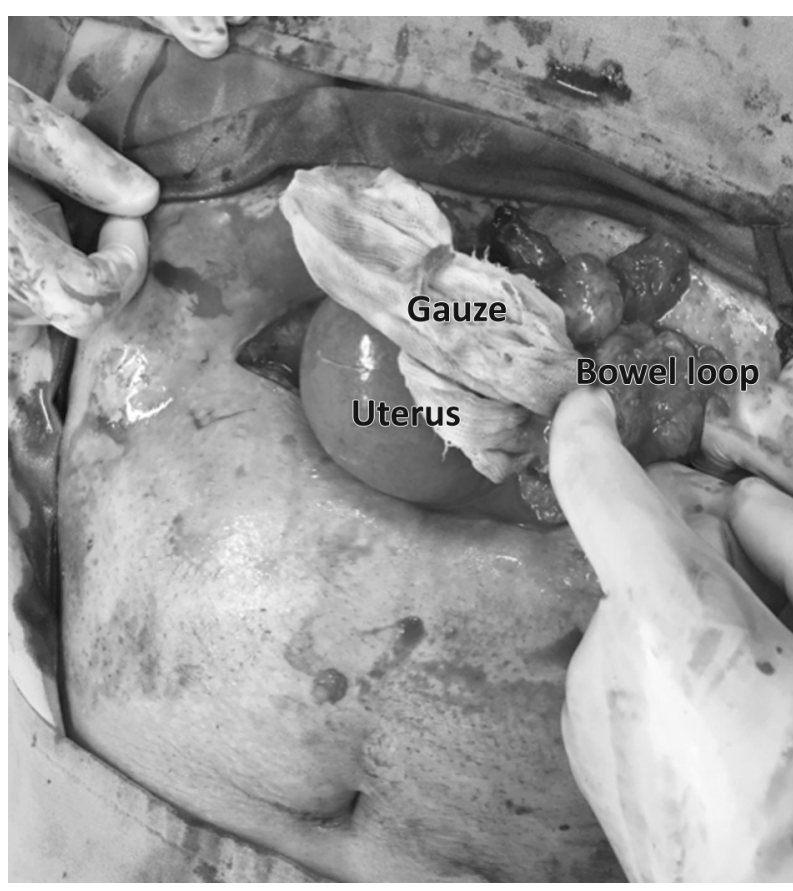

Figure 2: Intraoperative picture of retained foreign body

So, pre-operative diagnosis can be indeterminate. Usually it is seen that $75 \%$ of the gossypibioma are diagnosed only intraoperatively, similar to our case $^{7}$.

Surgical management is the definitive treatment. It involves adhesiolysis, drainage, and evacuation of abscess cavities, proximal diversion and resection anastomosis ${ }^{8}$. Complications like sepsis, intestinal obstruction, intra luminal transmigration, fistulization and perforation were not present in our case. Adhesiolysis and drainage of loculated collection followed by removal of gauze was performed.

The dictum "Prevention is always better than cure" holds true for gossypibioma as it is a very serious medicolegal issue. The heavy penalty for the surgeons is his/her years of earned reputation. Approximately $88 \%$ of cases occur in a situation where the sponge and instrument counts were declared "correct" 9 . So one needs to ensure proper count through experienced staffs, at least prior to and at the end of the procedure. Re-screening of high risk patients even if counts are documented correct will help in preventing such errors. If possible, use of surgical gauze sponges, which have been tagged with a radiofrequency identification (RFID) chip scanned with a barcode scanner can also help in early detection ${ }^{10}$. 


\section{Conclusion}

Gossypibioma, a preventable human error, can be decreased by improvement in patients care process and standardized count protocols. Though a rare entity, it should be considered as a differential diagnosis in patients presenting with pelvic mass. Subsequent surgical management will prevent severe morbidity and mortality in patients.

\section{References}

1. Rajput A, Loud P, Gibbs J, Kraybill W. Diagnostic challenges in patients with tumors. Journal of Clinical Oncology. 2013;21(19):3700-1.

2. Cima RR, Kollengode A, Garnatz J, Storsveen A, Weisbrod C, Deschamps C. Incidence and characteristics of potential and actual retained foreign object events in surgical patients. J Am Coll Surg 2008;207(1):80-7.

3. Alis H, Soylu A, Dolay K, Kalayci M, and Ciltas A. Surgical intervention may not always be required in gossypiboma with intraluminal migration. World J Gastroenterol. 2007;13(48): 6605-7.

4. Tsutsui JM, Armindo Hueb W, Alves Nascimento S, Borges Leal S, Andrade J, Mathias Jr W. Detection of retained surgical sponge by transthoracic and transesophageal echocardiography. J Am Soc Echocardiogr. 2003;16(11):1191-3.

5. Rajagopal A, Martin J. Gossypiboma “a surgeon's legacy": report of a case and review of the literature. Dis Colon Rectum. 2002;45(1):119-20.

6. Sun HS, Chen SL, Kuo CC, Wang SC, Kao YL. Gossypiboma - retained surgical sponge. J Chin Med Assoc. 2007;70(11):511-3.

7. Kopka L, Fischer U, Gross AJ, Funke M, Oestmann JW, Grabbe E. CT of retained surgical sponges (textilomas): pitfalls in detection and evaluation. J Comput Assist Tomogr.1996; 20(6):919-23.

8. Kaisar CW, Friedman S, Spurling KP, Slowick T, Kaiser HA. The Retained surgical sponge. Ann Surg. 1996;224(1):79-84.

9. Gawande AA, Studdert DM, Orav EJ, Brennan TA, Zinner MJ. Risk factors for retained instruments and sponges after surgery. N Engl J Med. 2003;348(3):229-35.

10. Macario, Morris D, Morris S. Initial clinical evaluation of a handheld device for detecting retained surgical gauze sponges using radiofrequency identification technology. Arch Surg. 2006;141(7):659-62. 